\title{
EVALUATION OF A NEW TYPE OF SEQUENTIAL LOT SIZING TECHNIQUES
}

\author{
Sven Axsäter and Björn Samuelsson \\ Luleå University of Technology, S-951 87 Luleă (Sweden)
}

\section{ABSTRACT}

The classical dynamic lot size problem without backlogging is usually solved with the aid of various heuristics. Most heuristics are sequential, i.e. the demand is considered period for period, and a decision whether to include the demand in a certain period in the preceding batch is taken without regarding the future demand. Recently, it has been shown how to design a sequential lot sizing rule that will optimize the av- erage performance, provided that a typical demand looks like a sequence of independent and identically distributed random numbers. The purpose of this paper is to evaluate if and how this methodology can be implemented in practice. The new lot sizing techniques are evaluated in a simulation study for different types of demand.

\section{INTRODUCTION}

This paper deals with the classical dynamic lot size problem without backlogging. In case of a finite horizon the optimal solution is easily obtained by applying the Wagner-Whitin algorithm [1]. This method is very seldom used in practice, though. One reason is that in most practical applications the horizon is infinite and the lot sizing problem is solved on a rolling horizon basis with a limited "forecast window". The Wagner-Whitin solution is quite sensitive with respect to the length of the "forecast window". Therefore, under such circumstances, it may be advantageous to use a simple heuristic (see [2]).

Most of the common heuristics are sequential techniques, i.e. the demand is considercd period for period, and a decision whether to include the demand in a certain period in the preceding batch is taken without regarding the demand beyond that period. Examples of such methods are the Silver-Meal heuristic [3] and Groff's method [4]. Sequential heuristics do not require a long horizon to work well and are therefore especially suitable in a rolling horizon environment.

When choosing lot sizing method this choice should, at least in principle, be based on some idea of what the demand looks like. If the demand can be characterized, it is usually easy to compare the performance of different methods. In some cases it may even be possible to determine the best lot sizing technique within a considered class of methods.

If a typical demand looks like a sequence of independent and identically distributed integral random numbers, Axsäter [5] provides a methodology for designing sequential lot sizing rules with optimal average performance. 
The purpose of this paper is to discuss if and how this methodology can be implemented in practice. In Section 2 we give a short summary of the theoretical background. Thereafter, in Section 3 we suggest an implementation framework that could be included in a practical inventory control system. This framework provides approximate decision rules based on an estimated demand distribution. Section 4 presents numerical results from a simulation study where the new lot sizing techniques are compared to the Silver-Meal heuristic. Finally in Section 5 we give some concluding remarks.

\section{SEQUENTIAL LOT SIZING WITH OPTIMAL AVERAGE PERFORMANCE}

We consider a traditional dynamic lot size problem without backlogging over an infinite horizon. There is a fixed set-up cost $S$. The linear holding cost $h$ is stationary and the end of period inventory is penalized.

When using a purely sequential lot sizing rule, the demand is considered period for period. In each step we decide whether to include the period demand in the preceding batch or to have a new set-up. Previous set-ups are known and can not be changed, and the decision is not based on the demand beyond the considered period.

In [5] it is assumed that a typical demand can be characterized as a sequence of independent and identically distributed random numbers with $p_{j}=$ probability that the period demand is equal to $j(j=0,1, \ldots)$. If the demand distribution is known, the sequential lot sizing problem is a simple Markov decision problem. We shall give a short summary of the solution of this problem. See [5] for details.

The state is completely characterized by the period number relative to the previous set-up. Let $V$ be the optimal expected average cost per period in the long run. Assume that the previous set-up is in period 1 and that we have already decided that there should be no set-ups in periods $2,3, \ldots, n$. Assume that we are using an optimal decision rule. If we decide to have a new set-up in period $n+1$ the expected cost per period over all future periods $n+1, n+2, \ldots$, is equal to $V$ by definition, since the demand beyond period $n+1$ is only known through its distribution. In case of no set-up the cost of satisfying the demand in period $n+1$ is $h n d_{n+1}$. Furthermore, it may be possible to reduce the cost beyond period $n+1$ relative to $V$. We denote the total expected cost reduction over periods $n+2, n+3, \ldots$ by $\delta_{n+1}$. Since we wish to maximize the total cost reduction, the optimal decision rule is to have a set-up in period $n+1$ if the demand $d_{n+1}$ satisfies the inequality.

$V-h n d_{n+1}+\delta_{n+1}<0$

We can also determine $\delta_{n}$ from $\delta_{n+1}$ with the aid of the dynamic programming recursion:

$\delta_{n}=\underset{d_{n+1}}{\mathrm{E}}\left[\max \left(0, V-h n d_{n+1}+\delta_{n+1}\right)\right]$

or equivalently

$\delta_{n}=\sum_{j=0}^{\left\lfloor\frac{\mathrm{V}+\delta_{n+1}}{\mathrm{hn}}\right]} p_{j}\left(V-h n j+\delta_{n+1}\right)$

where $[x]$ represents the integral part of $x$. In period 1 the cost increase is $S-V$ since the cost is $S$. This means that

$\delta_{1}=S-V$

Otherwise the expected cost per period could not be equal to $V$.

Denote by $N$ the smallest integer such that $N \geqslant S / h$. For $n \geqslant N$ there must be a set-up in pe$\operatorname{riod} n+1$ for any nonzero demand. But we will save $V$ in each period with zero demand. It is also evident that $\delta_{n}=\delta_{n+1}$ for $n \geqslant N$. Consequently $\delta_{N}=p_{0}\left(V+\delta_{N+1}\right)=p_{0}\left(V+\delta_{N}\right)$ and we have $\delta_{N}=V p_{0} /\left(1-p_{0}\right)$.

The optimal $V$ and $\delta_{n}: s$ that we need when applying the optimal decision rule (1) can easily be obtained from the following recursive procedure: 
1. Start with an initial $V^{0}$, e.g. $V^{0}=S$.

2. Given $V^{k}$, determine the resulting $\delta_{n}^{k}: s$ according to (3).

3. Next we determine $T_{n}^{k}$, the expected number of periods beyond period $n$ until the next set-up. Evidently $T_{N}^{k}=p_{0} /\left(1-p_{0}\right)$. Furthermore, it follows from (3) that

$T_{n}^{k}=\left(1+T_{n+1}^{k}\right) \sum_{j=0}^{\left[\frac{\nu^{k}+\delta_{n+1}^{k}}{h n}\right]} p_{j}$

4. We can now determine the resulting cost $V^{k+1}$ when using the $\delta_{n}^{k}: s$ in the decision rule (1).

$V^{k+1}=\frac{S+T_{1}^{k} V^{k}-\delta_{1}^{k}}{1+T_{1}^{k}}$

If $V^{k+1}>S-\delta_{1}^{k}+\epsilon$ set $k=k+1$ and go to 2 , otherwise stop. ( $S-\delta_{1}^{k}$ is a lower bound for the optimal $V$.)

It is shown in [5] that the procedure will converge monotonously to the optimal solution.

The determination of the optimal decision rule may also be somewhat simplified by noting that any demand $d \geqslant N$ will always lead to a set-up. Therefore for $d \geqslant N$ the costs are independent of the size of the demand. Consequently we can change the original probability distribution into

$$
\begin{aligned}
& p_{j}^{\prime}=p_{j} \quad j<N \\
& p_{N}^{\prime}=\sum_{j=N}^{\infty} p_{j}
\end{aligned}
$$

It is obvious that it is possible to improve the decisions if we look ahead, i.e. if we let the decisions be based also on the demand beyond the period considered for a set-up. In [5] the optimal decision rule is determined in the case when we look ahead one period. Since the computations involved are relatively complex a simpler approximate rule is also suggested. This rule is based on the $V$ and $\delta_{n}: s$ in the pure sequential case and suggests a set-up in period $n+1$ if

$$
\begin{aligned}
& V-S+\max \left\{0, V-h d_{n+2}+\delta_{2}\right\} \\
& \quad>V-h n d_{n+1}+\max \left\{0, V-h(n+1) d_{n+2}+\delta_{n+2}\right\}
\end{aligned}
$$

When applying (8) we evaluate the costs in period $n+1$ exactly, while the costs beyond period $n+1$ are evaluated as if the original purely sequential rule was used starting with period $n+2$. For a certain given demand distribution, the decisions will be improved compared to the original method. The decision rule $(8)$ is slightly more complex than (1), though. Both these rules are used in our numerical study.

\section{IMPLEMENTATION FRAMEWORK}

The two decision rules (1) and (8) are both based on a given probability distribution. Even if we in a practical application believe that a typical demand sequence will look like a sequence of independent random numbers, the probability distribution is not known and must be estimated in some way. Since this must be done for all individual items it is obvious that the estimation procedure must be very simple in order to be of practical interest. Furthermore, given the distributions, we need to determine the corresponding $V$ and $\delta_{n}: s$. If the demand distributions are updated continuously it would not be computationally feasible to go through these computations in each step for all items.

In order to simplify the computations we assume that the demand distribution is a negative binomial distribution. This is a two-parameter distribution and the probabilities $p_{j}$ can be determined from the average $m$ and the variance Var. Given $m$ and the coefficient of variation $k=\mathrm{Var} / m$ we have for $k>1$

$r=m /(k-1)$

$p=1 / k$

$p_{j}=\frac{r(r+1)(r+2) \ldots(r+j-1)}{j !} p^{r}(1-p)^{\prime}$

We shall only determine $V$ and the $\delta_{n}$ :s for 
integer values of the parameters $m$ and $k$, i.e. $m=0,1,2, \ldots$ and $k=0,1,2, \ldots$ Intermediate values are obtained by interpolation. For $m>0$ and $k>1$ we can use (9)-(11) for determining the required probabilities. If $m>0$ and $k=1$ the negative binomial distribution converges to a Poisson distribution, i.e.

$p_{j}=\frac{m^{j}}{j !} \mathrm{e}^{-m} \quad k=1$

If $k=0$ we have $p_{m}=1$ and all other probabilities are zero. If $m=k=0$ we obtain $V=0$ and $\delta_{n}=S$.

We assume that $V$ and the $\delta_{n}: s$ for nonnegative integer values of $m$ and $k$ have been tabulated in the lot sizing system. If we in our estimation obtain $m$ and $k$ such that

$i \leqslant m \leqslant i+1$

$j \leqslant k \leqslant j+1$

where $i$ and $j$ are integers, $V$ and the $\delta_{n}: s$ are obtained by interpolation according to

$$
\begin{aligned}
V= & (i+1-m)(j+1-k) V(i, j) \\
& +(i+1-m)(k-j) V(i, j+1) \\
& +(m-i)(j+1-k) V(i+1, j) \\
& +(m-i)(k-j) V(i+1, j+1) \\
& \cdot \\
\delta_{n}= & (i+1-m)(j+1-k) \delta_{n}(i, j) \\
& +(i+1-m)(k-j) \delta_{n}(i, j+1) \\
& +(m-i)(j+1-k) \delta_{n}(i+1, j) \\
& +(m-i)(k-j) \delta_{n}(i+1, j+1)
\end{aligned}
$$

When using this method we need separate tables for different values of $S / h$. But, in general, it would only be necessary to tabulate $V$ and the $\delta_{n}: s$ for a few values of $k$. It is also possible to reduce the tables by using a larger step for $m$.

In our numerical study we have based estimated averages and variances on all previous demand data. We have not used any data that are not utilized by the decision rules. This means that when applying (1) the estimate is based on the demand in the period considered for a set-up and all previous demands. Note that we are also including periods before the previous set-up. When applying ( 8 ) we utilize also the demand in the period following the period considered for a set-up. The average demand $m_{i}$ and the variance $\mathrm{Var}_{i}$ are updated recursively in the following way $\left(m_{1}=d_{1}\right.$, $\operatorname{Var}_{1}=0$ )

$m_{i}=\frac{i-1}{i} m_{i-1}+\frac{d_{i}}{i}$

$\operatorname{Var}_{i}=\frac{i-2}{i-1} \operatorname{Var}_{i-1}+\frac{1}{i}\left(d_{i}-m_{i-1}\right)^{2}$

In a practical case it may be possible to improve the estimates by using all demands in the "forecast window". It may also be more appropriate to reduce the influence of old data, for example, by using exponential smoothing when updating $m_{i}$ and $\operatorname{Var}_{i}$.

\section{NUMERICAL RESULTS}

In our numerical tests we have considered five types of demand. Four of these are independent random numbers as assumed in [5]. One is a deterministic sequence with "seasonal" variations. This demand sequence is used because we wish to evaluate how the considered methods perform in a case when the basic assumptions are not at all satisfied.

TABLE 1

Numerical tests: $S=20, h=1$

\begin{tabular}{rll}
\hline Case & Demand & $\begin{array}{l}\text { Number of } \\
\text { periods }\end{array}$ \\
\hline 1 & $p_{0}=0.5, p_{1}, p_{2}, \ldots, p_{10}=0.05$ & 25 \\
2 & $p_{0}=0.5, p_{1}, p_{2}, \ldots, p_{10}=0.05$ & 50 \\
3 & $p_{0}=0.25, p_{1}, p_{2}, \ldots, p_{10}=0.075$ & 25 \\
4 & $p_{0}=0.25, p_{1}, p_{2}, \ldots, p_{10}=0.075$ & 50 \\
5 & Poisson $\lambda=2$ & 25 \\
6 & Poisson $\lambda=2$ & 50 \\
7 & Poisson $\lambda=6$ & 25 \\
8 & Poisson $\lambda=6$ & 50 \\
9 & $d_{i}=4+2 \sin (2 \pi i / 50)$ & 25 \\
10 & $d_{i}=4+2 \sin (2 \pi i / 50)$ & 50 \\
\hline
\end{tabular}


TABLE 2

Costs per period and differences compared to the Silver-Meal heuristic. Standard deviation of differences in parenthesis

\begin{tabular}{|c|c|c|c|c|c|c|c|c|c|c|}
\hline & \multicolumn{2}{|c|}{ Case 1} & \multicolumn{2}{|l|}{ Case 2} & \multicolumn{2}{|l|}{ Case 3} & \multicolumn{2}{|l|}{ Case 4} & \multicolumn{2}{|l|}{ Case 5} \\
\hline & Cost & Diff. & Cost & Diff. & Cost & Diff. & Cost & Diff. & Cost & Diff. \\
\hline Sequential rule (1) & 6.44 & $\begin{array}{c}-0.11 \\
(0.02)\end{array}$ & 6.40 & $\begin{array}{c}-0.12 \\
(0.02)\end{array}$ & 8.78 & $\begin{array}{c}-0.04 \\
(0.02)\end{array}$ & 8.77 & $\begin{array}{c}-0.06 \\
(0.01)\end{array}$ & 7.10 & $\begin{array}{l}-0.08 \\
(0.01)\end{array}$ \\
\hline Look ahead rule (8) & 6.33 & $\begin{array}{c}-0.22 \\
(0.02)\end{array}$ & 6.30 & $\begin{array}{c}-0.22 \\
(0.02)\end{array}$ & 8.67 & $\begin{array}{c}-0.15 \\
(0.02)\end{array}$ & 8.66 & $\begin{array}{c}-0.17 \\
(0.01)\end{array}$ & 7.06 & $\begin{array}{c}-0.12 \\
(0.01)\end{array}$ \\
\hline \multirow[t]{3}{*}{ Silver-Meal } & 6.55 & - & 6.52 & - & 8.82 & - & 8.83 & - & 7.18 & - \\
\hline & \multicolumn{2}{|c|}{ Case 6} & \multicolumn{2}{|l|}{ Case 7} & \multicolumn{2}{|l|}{ Case 8} & \multicolumn{2}{|l|}{ Case 9} & \multicolumn{2}{|l|}{ Case 10} \\
\hline & Cost & Diff. & Cost & Diff. & Cost & Diff. & Cost & Diff. & Cost & Diff. \\
\hline Sequential rule (1) & 7.14 & $\begin{array}{c}-0.10 \\
(0.01)\end{array}$ & 11.88 & $\begin{array}{c}-0.02 \\
(0.01)\end{array}$ & 11.87 & $\begin{array}{c}-0.02 \\
(0.01)\end{array}$ & 10.73 & +0.13 & 10.62 & 0.11 \\
\hline Look ahead rule ( 8 ) & 7.10 & $\begin{array}{c}-0.14 \\
(0.01)\end{array}$ & 11.82 & $\begin{array}{c}-0.08 \\
(0.01)\end{array}$ & 11.81 & $\begin{array}{c}-0.08 \\
(0.01)\end{array}$ & 11.66 & 0 & 10.53 & 0.02 \\
\hline Silver-Meal & 7.24 & - & 11.90 & - & 11.89 & - & $\begin{array}{l}10.60 \\
11.66\end{array}$ & $\begin{array}{l}- \\
-\end{array}$ & $\begin{array}{l}10.51 \\
10.51\end{array}$ & $\begin{array}{l}- \\
-\end{array}$ \\
\hline
\end{tabular}

In all cases $S=20$ and $h=1$. We evaluate our methods over 25 periods as well as over 50 periods. This is because our lot sizing techniques can be expected to perform less well over a shorter horizon due to inaccurate estimates of the mean and variance. Table 1 describes all the tests. Cases 1-8 (with random demand) are simulated. When determining the per period cost in a simulation, periods at the end of the horizon that are not part of a full set-up cycle are excluded. In cases with 25 periods the results are based on 400 simulations and in cases with 50 periods on 200 simulations. The deterministic cases ( 9 and 10 ) are run just once. The horizon is extended beyond 25 or 50 periods until a joint set-up occurs.

Table 2 presents our results and those obtained when using the Silver-Meal heuristic. Note that in cases 9 and 10 we may get different results also with the Silver-Meal heuristic due to variations in the extension of the horizon. Both results are given.
The simple sequential rule (1) gives a cost reduction in all cases with independent stochastic demand variations (cases 1-8) varying between 0.2 and $1.8 \%$. In the two deterministic cases (9 and 10) with "seasonal" variations the cost increase is slightly above $1 \%$.

The look ahead rule $(8)$ is doing considerably better. In cases 1-8 the improvements are between 0.7 and $3.4 \%$. In cases 9 and 10 the results are almost equal to what is obtained when using the Silver-Meal heuristic.

The length of the horizon does not affect the results much but, in general, we get an improvement with a longer horizon.

\section{CONCLUSIONS}

Our evaluation of the new type of sequential lot sizing procedures shows that at least the look ahead rule could be expected to outperform the Silver-Mcal hcuristic under rela- 
tively general demand conditions. Our techniques would, on the other hand, require additional computational efforts and it would also be more difficult for a practitioner to understand the methodology.

Our methods may have a practical interest especially in rolling horizon situations when the lot sizing is very important and the "forecast window" is limited. In such cases our methods would probably also outperform the WagnerWhitin algorithm.

\section{REFERENCES}

1 Wagner, H.M. and Whitin, T.M., 1958. Dynamic version of the Economic lot size model. Manage. Sci., 5(1): 8996.

2 Blackburn, J.D. and Millen, R.A., 1980. Heuristic lot sizing performance in a rolling schedule environment. Dec. Sci., 11 (4): 691-701.

3 Silver, E.A. and Meal, H.C., 1973. A heuristic selecting lot size requirements for the case of a deterministic timevarying demand rate and discrete opportunities for replenishment. Prod. Inv. Manage., 14(2): 64-77.

4 Groff, G.K., 1979. A lot sizing rule for time-phased component demand. Prod. Inv. Manage., 20(4): 66-74.

5 Axsäter, S., 1988. A sequential lot sizing heuristic with optimal average performance. Manage. Sci., 34(11); 13241332. 